\title{
ИЗУЧЕНИЕ ДИНАМИЧЕСКОГО РАССЕЯНИЯ СВЕТА И ПОГЛОЩЕНИЯ УЛЬТРАЗВУКА В ВОДНЫХ РАСТВОРАХ 2,6-ДИМЕТИЛПИРИДИНА ВБЛИЗИ КРИТИЧЕСКОЙ ТОЧКИ ЖИДКОСТЬ-ЖИДКОСТЬ
}

\author{
К.Б. Эгамбердиев, С.3. Мирзаев \\ Институт ионно-плазменных и лазерных технологий АН РУз, ул. Дурмон йули, 33, \\ Ташкент, Узбекистан, kegamberdiev@yandex.ru
}

(Получена 21.06.2017)

\begin{abstract}
Ушбу ишда ишк̨ор галогенлар кўшилган, критик концентрацияга эга бўлган сувли 2,6-диметилпиридин аралашмасининг диффузия коэффициенти ва ультратовуш ютилиш коэффициенти ўлчанган. Корреляция узунлигининг ва флуктуация релаксация тезлигининг критик даражалари аникеланиб, ажралиш критик нуқттаси яқ̆ннда учинчи компонентнинг хосса параметрларига сезиларли таъсири кўрсатилган.
\end{abstract}

Коэффициенты диффузии и поглощения ультразвука критического состава водных растворов 2,6-диметилпиридина с критической концентрацией были измерены без и с добавлением солей. Определены критический показатель длины корреляции флуктуации и скорость релаксации флуктуации, показано существенное влияние третьего компонента на поведение параметров вблизи критической точки расслаивания.

\begin{abstract}
The diffusion coefficient and ultrasonic attenuation of 2,6-dimethylpyridine-water mixtures of critical composition have been measured without and with alkali halides added. The critical exponent of the fluctuation correlation length and the critical exponent of the relaxation rate of the fluctuations have been evaluated to show noticeable influences from the third components.
\end{abstract}

\section{I. ВВЕДЕНИЕ}

В последние годы большое внимание в исследованиях свойств жидкостей было уделено критическим явлениям. Это обусловлено тем, что вблизи критической точки расслаивания бинарных жидкостей локальные флуктуации концентрации вызывают аномалии в термодинамических и транспортных параметрах. На основе концепции универсальности скейлинга (масштабирования) и перенормировки [1] были предложены теоретические модели критической динамики $[2,3]$, в частности, для описания поведения жидкости в области критической точки подходит модель динамического скейлинга $[4,5]$ и связанных мод [6, 7]. Современные модели включают функцию кроссовера, представляющую переход от универсального изинговского поведения (асимптотически близкого к критической точке) к неуниверсальному поведению среднего поля вдали от критической области.

Эффект кроссовера может отражать элементарные химические реакции, такие как структурная изомеризация молекул, димеризация и образование мицелл. Теоретические модели позволяют утверждать, что химические реакции между двумя компонентами бинарных жидкостей замедляются при приближении к критической точке $[8,9]$, но при этом рассматривается только система мономердимер. Как было показано в [10], спектры ультразвукового поглощения критическим составом изомасляной кислоты и воды показывают существенные 
эффекты замедления вблизи критической температуры не только в равновесии мономера с линейным димером карбоновой кислоты, но также и в системе линейный димер-циклический димер, причем последний по существу является мономолекулярным. Поэтому в настоящее время не существует единого взгляда на взаимосвязь между критическими флуктуациями концентрации и стехиометрически определенными элементарными реакциями.

В работе [11] было показано, что при проведении ультразвукового спектроскопического исследования критической системы 2,6-диметилпиридинвода (2,6-ДМП-Н $2 \mathrm{O})$ наблюдалась более низкая критическая точка расслаивания при $T_{c}=306.83 \mathrm{~K}$ с критической массовой долей 2,6-ДМП $Y_{c}=0.2913$ [12]. Кроме того, поскольку 2,6-ДМП является слабым основанием, протолиз может иметь место в водных растворах, и по аналогии с упаковкой стопки (стекинг) циклических пуриновых оснований может быть предложено образование агрегатов 2,6-ДМП. Изучение диэлектрической и ультразвуковой релаксации, а также деполяризованное рэлеевское рассеяние и ЯМР-исследования подтвердили идею агрегатов 2,6-ДМП, включая воду [13-15]. Следовательно, ультразвуковые спектры смесей 2,6-ДМП- $\mathrm{H}_{2} \mathrm{O}$, кроме вкладов от флуктуаций концентрации, должны обладать богатым спектром, обусловленным элементарными химическими равновесиями. Другим преимуществом системы 2,6-ДМП- $\mathrm{H}_{2} \mathrm{O}$ для исследования является наличие данных по теплоемкости, сдвиговой вязкости и динамическому рассеянию света [12], которые значительно облегчают оценку флуктуационного вклада в спектрах ультразвукового поглощения. В этой работе коэффициент поглощения ультразвука в широком частотном диапазоне и коэффициенты диффузии критического состава водных растворов 2,6-ДМП с критической концентрацией были измерены без и с добавлением галогенидов щелочных металлов. Данные были проанализированы с точки зрения поведения степенного закона.

\section{II. МЕТОДИКА И ЭКСПЕРИМЕНТ}

Коэффициенты диффузии $D$ смесей критического состава были определены с помощью метода динамического рассеяния света - фотонной корреляционной спектроскопии [16]. Сущность метода состоит в том, что свет, рассеянный исследуемым участком образца, направляется на фотокатод квадратичного по полю фотоприемника. На катоде возникают биения между различными частотными компонентами спектра падающего света. При этом освещенность фотокатода и, следовательно, фототок оказываются промодулированы по амплитуде частотами биений (от нуля до ширины спектра рассеяния). Образцы освещали удвоенной частотой Nd YAG лазера ( $\lambda_{0}=532$ нм, Coherent, SantaClara, CA, CША; $\lambda_{0}-$ длина волны в вакууме). Часть рассеянного света при угле $\pi / 2$ была зарегистрирована с помощью ФЭУ (Hamamatsu R647P, Хамамацу, Япония) и проанализирована в реальном времени платой цифровой корреляции (ALV-5000/E, Langen, Германия). Плата была способна обеспечить нормированную интенсивность фотонов для автокорреляционной функции $S\left(q_{r}, t\right)$ в течение периода от $2 \times 10^{-7}$ до $3.4 \times 10^{3}$ с [17, 18]. Здесь

$$
q_{r}=\frac{4 \pi n}{\lambda_{0}} \sin (\Theta / 2)
$$


абсолютное значение волнового вектора при выбранном угле рассеяния $\Theta=\Theta_{r}=\pi / 2$. В ур-ии (1) параметр $n$ является оптическим показателем преломления, который измерен с помощью рефрактометра (Zeiss, Obercochem, Германия).

Коэффициенты диффузии

$$
D(T)=\left[q_{r}^{2} \tau_{c}\left(q_{r}, T\right)\right]^{-1}
$$

были получены из времен корреляции $\tau_{c}$ функции автокорреляции фотона $g^{(1)}$ рассеянного света. Самобиение частот рассеянного света связано с нормированной автокорреляционной функцией $g^{(2)}\left(\tau_{c}\right)$ интенсивности в соответствии с соотношением

$$
\left|g^{(1)}\left(\tau_{c}\right)\right|^{2}=g^{(2)}\left(\tau_{c}\right)-1 .
$$

Рэлеевское рассеяние света в жидкостях дает форму лоренциана, показывающую экспоненциальное затухание функции корреляции времени в соответствующей частотной области

$$
S\left(q_{r}, t\right)\left(g^{(2)}\left(\tau_{c}\right)-1\right)=\exp \left[-t / \tau_{c}\left(q_{r}, T\right)\right] .
$$

Уравнение (4) представляет собой мономодальную, строго экспоненциально спадающую корреляционную функцию. Отклонения от строго экспоненциального поведения преимущественно наблюдались вдали и в области, близкой к критической температуре $T_{c}$. Коэффициенты диффузии, таким образом, рассчитывались из основных времён затухания $\tau_{c}$ функции распределения.

В экспериментах по квазиупругому рассеянию света ячейка с исследуемым образцом была снабжена оболочкой для циркуляции термостатирующей жидкости. Спектрометр был установлен на закрепленном вибрационно-демпфированном оптическом столе. Ячейка с исследуемым образцом помещена в дополнительную термостатирующую систему, где циркулировала жидкость с постоянной температурой, что обеспечивало высокую стабильность температуры во время измерений. Колебания и градиенты температуры внутри ячейки были меньше, чем 0.01 К. С помощью термометра Pt-100, расположенного в измеряемой ячейке, $T$ измерена с точностью 0.01 К. Небольшие изменения в температуре и разброс времен корреляции $\tau_{c}$ привели к экспериментальной погрешности $\Delta D / D \leq 5 \%$ в измерениях коэффициента взаимной диффузии.

\section{III. КОЭФФИЦИЕНТ ВЗАИМНОЙ ДИФФУЗИИ}

Коэффициенты диффузии $D$ для смесей критического состава приведены в зависимости от приведенной температуры $\varepsilon$ на рис. 1. Значения $D$ рассчитаны в соответствии с формулой (2) из времени корреляции $\tau_{c}$, как следует из экспериментов по квазиупругому рассеянию света. На рис. 1 в двойном логарифмическом представлении каждая серия данных отображает линейную часть, отражающую поведение степенного закона, и она показывает изгиб при приближении к критической температуре, т.е. когда $\varepsilon$ стремится к 0 . Значения $D$ смеси 2,6-ДМП $+\mathrm{H}_{2} \mathrm{O}$ без соли также отклоняются при $\varepsilon>0.03$. Было зафиксировано заметное отклонение данных параметров при более высоком содержании соли [19].

Пренебрегая корректировкой кроссовера при $\varepsilon>10^{-3}$, линейная часть соотношения $\log (D)$ в зависимости от $\log (\varepsilon)$ может быть представлена выражением для критического вклада [20, 21]: 


$$
D_{c}=\frac{k_{B} T}{6 \pi \eta_{s} \xi}=\frac{k_{B} T}{6 \pi \eta_{b} Q_{0}^{Z_{\eta}} \xi_{0}^{1+Z_{\eta}} \varepsilon^{-v\left(1+Z_{\eta}\right)}}=D_{0}^{v *}
$$

к коэффициенту диффузии. В этом уравнении $k_{\mathrm{B}}$ - постоянная Больцмана, $v^{*}-$ критический показатель, $\eta_{s}-$ общая вязкость, $\eta_{b}-$ фоновая вязкость вдали от критической точки.

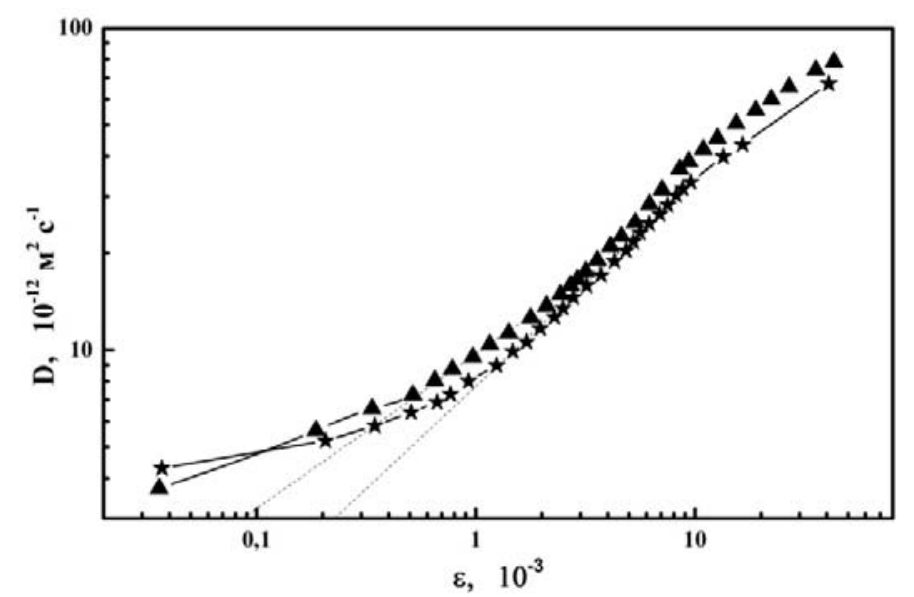

Рис. 1. График в двойном логарифмическом масштабе коэффициента взаимной диффузии $D$ (ур-ие 5) в зависимости от приведенной температуры $\varepsilon$ для двух смесей 2,6-ДМП+ $\mathrm{H}_{2} \mathrm{O}$ критического состава с 1\% (вес/вес) добавленной соли: $\mathrm{KBr}(\boldsymbol{\Delta}), \mathrm{NaBr}(\star)$. Пунктирные линии обозначают продолжение линейных частей на графиках.

Часто подобная зависимость $D_{c}$ от $T$, а также температурная зависимость в фоновой вязкости не рассматриваются, так как интерес представляет только небольшой диапазон температур вблизи $T_{c}$, где доминирует поведение степенного закона. В этом случае

$$
v^{*}=\left(1+Z_{\eta}\right) v
$$

принимается как сочетание универсального показателя $Z_{\eta}$ сдвиговой вязкости и критического показателя $v$ длины корреляции флуктуации. Значения $v^{*}$, полученные из данных коэффициента диффузии, приведены в табл. 1.

Общие характеристики зависимостей коэффициента диффузии при приведённой температуре напоминают теоретическую масштабную функцию [22]

$$
D=D_{c}\left[R_{D} K(q \xi)\left\{1+\frac{q \xi}{4}\right\}^{Z_{\eta}}+\left\{\frac{3 \pi}{8} \frac{\eta_{s}}{\eta_{b}}\right\}\left\{1+(q \xi)^{2}\right\} /\left(q_{c} \xi\right)\right],
$$

которая включает в себя эффекты кроссовера. В уравнении (7) $D_{c}$ является выражением Кавасаки-Феррелл для коэффициента диффузии (ур-ние 5), $R_{D}=1.05$ $[23,24])$ - отношение универсальных амплитуд, $K(q \xi)$ - функция Кавасаки [25], $\xi$ длина корреляции флуктуации, $q=q_{r}-$ волновое число (ур-ние 1) и $q_{c}-$ критическое волновое число как в уравнении (1). Несмотря на общее согласие экспериментальных данных для $D$ с формой теоретического отношения, мы предполагаем, что их изгиб при приближении к $T_{c}(\varepsilon \rightarrow 0)$, также как в случае сдвиговой вязкости, отражает некритические процессы. 
Таблица 1. Показатели оптического преломления $n$, критические показатели $y_{\eta}$ и $v^{*}$ (ур-ие 5) для 2,6-ДМП+ $\mathrm{H}_{2} \mathrm{O}+$ соль смеси критического состава.

\begin{tabular}{c|c|c|c|c}
\hline Соль & $Y_{\text {соль }}$ & $n$ & $y_{\eta} \pm 0.006$ & $v^{*} \pm 0.03$ \\
\hline- & 0 & 1.407 & 0.044 & 0.66 \\
$\mathrm{NaF}$ & 0.005 & 1,393 & 0.039 & 0.57 \\
$\mathrm{NaCl}$ & 0.003 & $1.391[3]$ & 0.045 & 0.60 \\
$\mathrm{NaCl}$ & 0.004 & 1.387 & 0.044 & 0.58 \\
$\mathrm{NaCl}$ & 0.005 & $1.381[32]$ & 0.042 & 0.53 \\
$\mathrm{NaBr}$ & 0.005 & 1.419 & 0.049 & 0.53 \\
$\mathrm{NaBr}$ & 0.01 & 1.389 & 0.053 & 0.52 \\
$\mathrm{NaBr}[19]$ & 0.015 & 1.404 & 0.035 & 0.49 \\
$\mathrm{NaBr}[19]$ & 0.03 & 1.409 & 0.029 & 0.48 \\
$\mathrm{NaI}$ & 0.005 & 1.390 & 0.052 & 0.60 \\
$\mathrm{NaI}$ & 0.01 & 1.389 & 0.059 & 0.58 \\
$\mathrm{LiBr}$ & 0.01 & 1.388 & 0.043 & 0.58 \\
$\mathrm{NaBr}$ & 0.01 & 1.389 & 0.053 & 0.52 \\
$\mathrm{KBr}$ & 0.01 & 1.389 & 0.044 & 0.61 \\
\hline
\end{tabular}

Для подтверждения этого спектры избыточного поглощения ультразвука

$$
(\alpha \lambda)_{\text {изб }}(f)=\alpha \lambda-B f
$$

системы 2,6-ДМП+ $\mathrm{H}_{2} \mathrm{O}$ представлены на рис. 2. В ур-нии (8) $\alpha$ является коэффици-

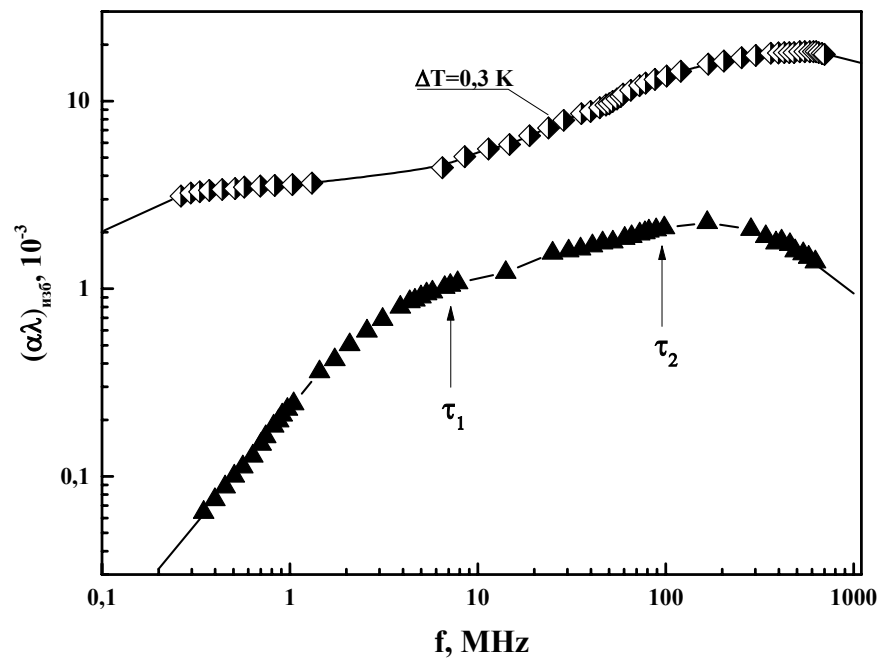

Рис. 2. Спектры избыточного поглощения ультразвука для смеси критического состава 2,6диметилпиридин-вода (открытые символы) при $\Delta T=T_{c}-T=0.3 \mathrm{~K}(*)$ [28]. Также показаны спектры избыточного поглощения для 2,6-ДМП $+\mathrm{H}_{2} \mathrm{O}$ в смесях некритического состава $\mathrm{Y}_{2,6-\text {-дмп }}=0.148$ ( $\left.\mathbf{\Delta}\right)$ при $25^{\circ} \mathrm{C}[11]$. Стрелками показаны времена релаксации.

ентом поглощения ультразвука, $\lambda$ - длина волны звука в жидкости, коэффициент $B$ - асимптотический член высокой частоты, который здесь не представляет интереса и вычтен из поглощения на единицу длины волны. Спектр для смеси критического состава диметилпиридин-вода при температуре, близкой к $T_{c}(\Delta T=0.3 \mathrm{~K})$ показывает значительный вклад критической флуктуации в широком диапазоне 
частот (рис. 2). На значительном расстоянии от $T_{c}$ в результате уменьшения влияния эффекта от критических флуктуаций $[19,26,27]$ существенно уменьшается поглощение при низких частотах $(f<5$ МГц). Однако на высоких частотах $(f>$ 5 МГц) значения $\alpha \lambda$ мало меняются, что указывает на заметный вклад некритических релаксационных процессов в этом частотном диапазоне. Соответственно, избыточное поглощение присутствует также в высокочастотной

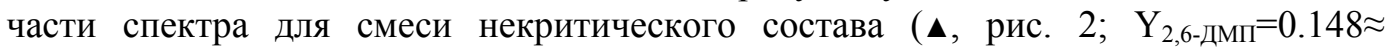

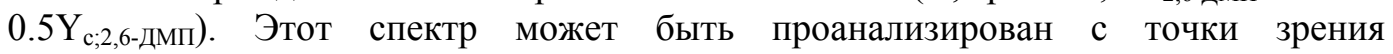
спектральных функций типа двух кривых дебаевской релаксации с временами релаксации, обозначенными стрелками на рис. 2. Вариацией $\mathrm{pH}$ некритического состава четко показано, что высокочастотный дебаевский вклад отражает реакцию протолиза. Некритический вклад при более низких частотах с частотой релаксации около 10 МГц в спектре рис. 2 соответствует равновесию мезоскопических агрегатов 2,6-диметилпиридина, вероятно, по схеме реакции

$$
(2,6-Д М П)_{n}+2,6-Д М П \leftrightarrow(2,6-Д М П)_{n+1}, \quad n=1,2,3, \ldots
$$

Агрегация диметилпиридина может привести к уменьшению гидрофобного взаимодействия с водой. Возможно, она также сопровождается связыванием двух молекул диметилпиридина с помощью молекулы воды через водородные связи между неподеленной парой электронов диметилпиридина, азота и положительно заряженного участка водорода воды.

Отметим, что агрегация молекул также играет существенную роль в критических составах 3-метилпиридина (таких как 3-MП+ $\mathrm{H}_{2} \mathrm{O}+\mathrm{NaBr}[29,30]$ и 3-MП $+\mathrm{D}_{2} \mathrm{O}+\mathrm{NaBPh}_{4}$ ) [31]. В этих смесях были зарегистрированы стабильные мезоскопические капли размером порядка 0.1 мкм [23]. Таким образом, образование агрегатов способствует предотвращению дальнейшего роста локальных флуктуаций концентрации при приближении к критической температуре и тем самым задержке увеличения вязкости, а также уменьшению коэффициента диффузии.

Несмотря на наши ожидания изингоподобного критического поведения в расслаивании для смесей, в данном исследовании только система 2,6-ДМП+ $\mathrm{H}_{2} \mathrm{O}$ без добавления соли (табл. 1) показывает критическое показатели близкие к $v^{*}=$ $\left(1+Z_{\eta}\right) v=0.673$ и $y_{\eta}=v Z_{\eta}=0.0435$, совпадающие с общепринятыми теоретическими значениями для $v$ и $Z_{\eta}$. Значение $v^{*}$ для смесей с добавлением соли меньше, чем $v^{*}\left(Y_{\text {соль }}=0\right)=0.66$ (табл. 2). Значения $y_{\eta}$ смесей с добавлением $\mathrm{NaCl}, \mathrm{NaBr}, \mathrm{NaI}$, увеличиваются с концентрацией соли, но, как правило, уменьшается $y_{\eta}\left(Y_{\text {соль }}=0\right)$, равное 0.044 при более высоких $Y_{\text {соль }}$. С примесью фторида натрия $y_{\eta}\left(Y_{\mathrm{NaF}}=0.005\right)<$ 0.044 , а в системах, содержащих $\mathrm{LiBr}$ и $\mathrm{KBr}$, значение $y_{\eta} \approx 0.044\left(Y_{\text {соль }}=0.01\right)$.

Критический показатель длины корреляции и показатель сдвиговой вязкости выражается как:

$$
v=v^{*}-y_{\eta}
$$

а также

$$
Z_{\eta}=y_{\eta} / v,
$$

соответственно, что представлено в табл. 2. Для всех галогенидов щелочных металлов наблюдалось сначала уменьшение значений $v$ с $Y_{\text {соль }}$. Начальные наклоны практически идентичны с растворами, содержащими $\mathrm{NaCl}$ и $\mathrm{NaBr}$. Они меньше для растворов, содержащих $\mathrm{NaF}$ и $\mathrm{NaI}$. По мере увеличения содержания $\mathrm{NaBr}, v$ падает 
до значения 0.45, которое является наименьшим для критических составов в данном исследовании. В смеси 2,6-диметилпиридин-вода, содержащей ионную жидкость 1-бутил-3-метилимидазолий гексафторфосфата $(M=419.36$ г/моль) в качестве добавки, было отмечено падение критического показателя длины корреляции до $v=0.39$ при массовой доле ионной жидкости $Y_{\text {иж }}=0.015$, но с дальнейшим ростом до значений: $v=0.59$ при $Y_{\text {иж }}=0.03$ и $v=0.69$ при $Y_{\text {иж }}=0.06$ [19]. Это различное поведение может быть связано со значительным размером органических ионов, которые влияют на критические флуктуации из-за их большого объема.

Таблица 2. Критический показатель $v$ (в уравнении 9), флуктуации длины корреляции и показатель вязкости $Z_{\eta}$ (в уравнении 10$), \tilde{v}$ - показатель скорости релаксации флуктуаций (в уравнении 11) для 2,6-ДМП+ $\mathrm{H}_{2} \mathrm{O}+$ смеси солей критических составов.

\begin{tabular}{c|c|c|c|c}
\hline Соль & $Y_{\text {соль }}$ & $\nu \pm 0.04$ & $Z_{\eta} \pm 0.015$ & $\tilde{v} \pm 0.15$ \\
\hline- & 0 & 0.62 & 0.071 & 1.90 \\
$\mathrm{NaF}$ & 0.005 & 0.53 & 0.074 & 1.73 \\
$\mathrm{NaCl}$ & 0.003 & 0.55 & 0.082 & 1,92 \\
$\mathrm{NaCl}$ & 0.004 & 0.54 & 0.081 & 1,71 \\
$\mathrm{NaCl}$ & 0.005 & 0.49 & 0.086 & 1,76 \\
$\mathrm{NaBr}$ & 0.005 & 0.48 & 0.102 & 1.74 \\
$\mathrm{NaBr}$ & 0.01 & 0.47 & 0.113 & 1.79 \\
$\mathrm{NaBr}[19]$ & 0.015 & 0.45 & 0.078 & 1.77 \\
$\mathrm{NaBr}[19]$ & 0.03 & 0.45 & 0.064 & 1.74 \\
$\mathrm{NaI}$ & 0.005 & 0.55 & 0.095 & 1.85 \\
$\mathrm{NaI}$ & 0.01 & 0.52 & 0.113 & 1.62 \\
$\mathrm{LiBr}$ & 0.01 & 0.54 & 0.080 & 2.07 \\
$\mathrm{NaBr}$ & 0.01 & 0.47 & 0.117 & 1.79 \\
$\mathrm{KBr}$ & 0.01 & 0.57 & 0.078 & 1.82 \\
\hline
\end{tabular}

Во всех щелочно-галоидных смесях критический показатель вязкости возрастает с увеличением содержания соли до $Z_{\eta}=0.115 \pm 0.015$ при $Y_{\mathrm{NaBr}}$ и $Y_{\mathrm{Nal}}=0.01$. Сначала их показатель для вязкости аналогичным образом увеличивается до $Z_{\eta}=0.090$ при $Y_{\text {иж }}=0.015$, а затем, при более высоком содержании соли, он показывает почти постоянное значение $\left(Z_{\eta}=0.088\right.$ при $Y_{\text {иж }}=0.06$ [19]), что еще раз доказывает существование определенной роли объема ионов в дополнение к напряженности электрического поля, что необходимо учитывать в их взаимовлиянии с критической динамикой.

Критические показатели $v$ и $Z_{\eta}$ можно рассматривать комбинированными в зависимости:

$$
\Gamma=\Gamma_{0} \varepsilon^{\tilde{v}},
$$

где скорость релаксации флуктуаций параметра порядка

$$
\Gamma=2 D \xi^{-2}
$$

при приведённой температуре. Сосредоточивая внимание на температурном диапазоне, в котором эффекты кроссовера незначительны, можно $D$ заменить на $D_{c}$, и таким образом в формуле (5) исключить длину корреляции флуктуаций

$$
\Gamma=72 \pi^{2} \eta_{b}^{2} D_{0}^{3}\left(Q_{0} \xi_{0}\right)^{2 Z_{\eta}} \varepsilon^{\eta\left(3+Z_{\eta}\right)} /\left(k_{B} T\right)^{2}=\Gamma_{0} \varepsilon^{\tilde{v}}
$$


с $\tilde{v}=v\left(3+Z_{\eta}\right)$. Это соотношение с амплитудой $\Gamma_{0}$ не зависит от температуры и соблюдается лишь в небольшом диапазоне температур вблизи $T_{c}$. Для измерений в более широком температурном диапазоне существенна явная зависимость скорости релаксации от $\varepsilon$ (рис. 3), а также значительная температурная зависимость фоновой вязкости (рис. 3, вставка). Таким образом, на рис. 3 приведены экспериментальные данные для $\Gamma$ в зависимости от состава смеси, и мы определяем из экспериментальных данных $\eta_{s}$ и $D$ значения расчетных критических показателей $\Gamma$ различных составов.

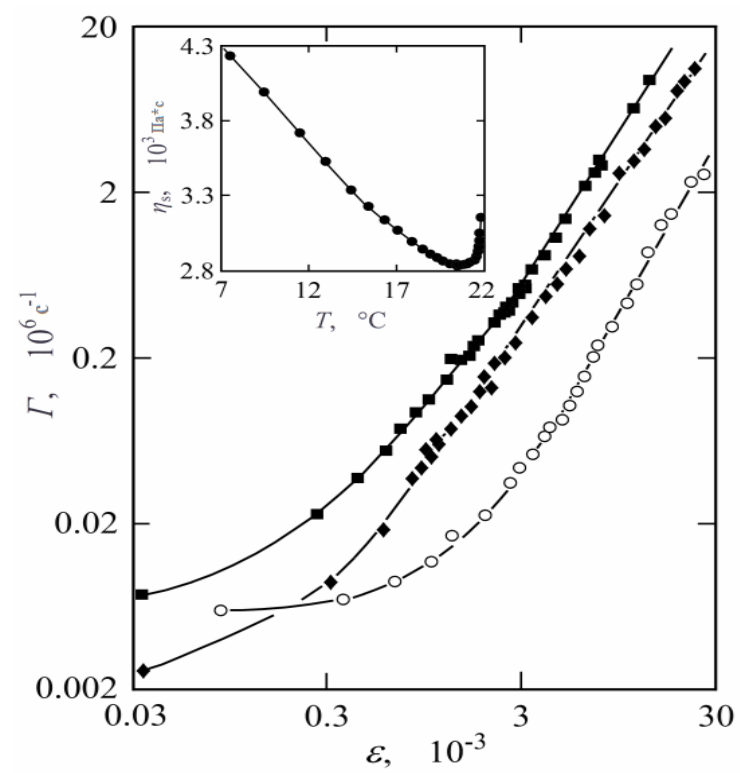

Рис. 3. Скорость релаксации Г флуктуаций концентрации в зависимости от приведённой температуры $\varepsilon$ для 2,6-ДМП $+\mathrm{H}_{2} \mathrm{O}$ смесей критического состава, содержащих некоторые ионные добавки: $\mathrm{NaBr}(\boldsymbol{\bullet}), \mathrm{Y}_{\text {соль }}=0.1 ; \mathrm{NaF}(\bullet), \mathrm{Y}_{\text {соль }}=0.005$; ионная жидкость 1-бутил-3метилимидазоли бис (трифторметил-сульфонил) имид (о) [19]. На вставке показана сдвиговая вязкость $\eta_{s}$ для одной из смесей, $\mathrm{Y}_{\mathrm{NaF}}=0.005(\bullet)$, как функция температуры $T$.

\section{IV. ЗАКЛЮЧЕНИЕ}

Критический показатель длины корреляции флуктуации $v$ и критический показатель скорости релаксации флуктуаций $\tilde{v}$ для смесей критического состава 2,6-диметилпиридин-вода хорошо согласуются с их теоретическими предсказаниями значений $0.63,0.069$ и 1.3 соответственно. Однако добавление щелочных галогенидов изменяет значения критических показателей, то есть при добавлении солей критический показатель длины корреляции флуктуации уменьшается до $25 \%$, а критический показатель скорости релаксации флуктуаций уменьшается до 10\%. Изменения критических показателей особенно сильны при малых массовых долях $Y_{\text {соль }}$ соли. При $Y_{\text {соль }}>0.005$ критический показатель стремится к постоянному значению. Такое поведение соответствует изменению критических показателей системы 2,6-ДМП+ $\mathrm{H}_{2} \mathrm{O}$, когда добавляется более 1.5 массовой доли ионной жидкости [19]. 
Исследования динамического рассеяния света были проведены в физическом факультете Гёттингенского университета (Германия), и авторы выражают благодарность У. Каатце за участие в обсуждении результатов, а также международным фондам ДААД (Бонн, Германия) и Александра фон Гумбольдта за поддержку.

Работа выполнялась в рамках гранта № МУ-Ф3-2017101937 программы фундаментальных исследований Академии наук Узбекистана.

\section{ЛИТЕРАТУРА}

1. H.E. Stanley, Rev. Mod. Phys. 71, 5358(1999).

2. M.A. Anisimov, Critical Phenomena in Liquids and Liquid Crystals, Gordon and Breach, London (1991).

3. A. Onuki, Phase Transition Dynamics, Cambridge University Press, Cambridge (2002).

4. R.A. Ferrell, J.K. Bhattacharjee, Phys. Rev. A 31, 788 (1985).

5. J.K. Bhattacharjee, R.A. Ferrell, Phys. Rev. A 250, 83 (1998).

6. R. Folk, G. Moser, Phys. Rev. E 57, 705 (1998).

7. R. Folk, G. Moser, Phys. Rev. E 58, 6246 (1998).

8. I. Procaccia, M. Gittermann, Phys. Rev. Lett. 46, 1163 (1981).

9. H.G.E. Hentschel, I. Procaccia, J. Chem. Phys. 76, 666 (1982).

10. U. Kaatze, S.Z. Mirzaev, J. Phys. Chem. A 104, 5430 (2000).

11. S.Z. Mirzaev, I. Iwanowski, M. Zaitdinov, U. Kaatze, Chem. Phys. Lett. 431, 308 (2006).

12. S.Z. Mirzaev, R. Behrends, T. Heimburg, J. Haller, U. Kaatze, J. Chem. Phys. 124, 144517 (2006).

13. U. Kaatze, D. Woermann, J. Phys. Chem. 88, 284 (1984).

14. N. Ito, T. Kato, J. Phys. Chem. 88, 801 (1984).

15. U. Kaatze, U. Schreiber, Chem. Phys. Lett. 148, 241 (1988).

16. C. Trachimow, L. De Maeyer, U. Kaatze, J. Phys. Chem. B 2, 4483 (1998).

17. K. Schätzel, Appl. Phys. B 42, 193 (1987).

18. K. Schätzel, M. Drewel, S. Stimac, J. Mod. Opt. 35, 711 (1988).

19. D. Truszkowska, M. Dzida, U. Kaatze, Chem. Phys. Lett. 604, 53 (2014).

20. K. Kawasaki, Ann. Phys. (N.Y.) 61, 1 (1970).

21. R.A. Ferrell, Phys. Rev. Lett. 24, 1169 (1970).

22. H.C. Burstyn, J.V. Sengers, J.K. Bhattacharjee, R.A. Ferrell, Phys. Rev. A 28, 1567 (1983).

23. J. Leys, D. Subramanian, E. Rodezno, B. Hammouda, M.A. Anisimov, Soft Matter 9, 9326 (2013).

24. S. Das, J.V.M.E. Sengers, J. Chem. Phys. 127, 144506 (2007).

25. C. Domb; M.S. Green. Phase Transitions and Critical Phenomena (Academic Press, New York, 6, 1976).

26. M.A. Anisimov. Critical Phenomena in Liquids and Liquid Crystals (Gordon and Breach, London, 1991).

27. J.K. Bhattacharjee, U. Kaatze, S.Z. Mirzaev, Rep. Prog. Phys. 73, 066601 (2010).

28. U. Kaatze, U. Schreiber, Chem. Phys. Lett. 148, 241 (1988).

29. A.F. Kostko, M.A. Anisimov, J.V. Sengers, Phys. Rev. E 70, 026118 (2004).

30. M.-P. Hernández, F. Monroy, F. Ortega, R.G. Rubio. J. Chem. Phys. 122, 104501 (2005).

31. J. Leys, D. Subramanian, E. Rodezno, B. Hammouda, M.A. Anisimov, Soft Matter 9, 9326 (2013).

32. K.G. Wilson, Rev. Mod. Phys. 55, 583 (1983). 